\title{
Stirring of the northeast Atlantic spring bloom: A Lagrangian analysis based on multisatellite data
}

\author{
Y. Lehahn, ${ }^{1,2}$ F. d'Ovidio, ${ }^{3}$ M. Lévy, ${ }^{1}$ and E. Heifetz ${ }^{2}$ \\ Received 7 September 2006; revised 26 January 2007; accepted 16 May 2007; published 4 August 2007.
}

[1] The effect of the geostrophic stirring on phytoplankton variability during the northeast Atlantic spring bloom is studied by analyzing satellite derived surface chlorophyll, sea surface temperature, and sea surface height. The calculation of unstable manifolds is used as a diagnostic of the transport properties of the geostrophic velocity field (calculated from the sea surface height). We identify two mechanisms by which the geostrophic velocity field acts on chlorophyll patterns. The first mechanism is a direct effect of the horizontal transport on already formed chlorophyll. By acting as "sticking" transport barriers, the unstable manifolds are shown to (1) modulate the fronts of already formed phytoplankton in lobular structures, (2) create spiralling chlorophyll anomalies within eddies, and (3) produce chlorophyll filaments. The second mechanism is an indirect effect on in situ chlorophyll production mediated by nutrient upwelling. Supported by a recent study on the vertical velocities of the northeast Atlantic (Legal et al., 2006), we argue that the horizontal unstable manifolds also shape the filamentary, vertical velocity cells, and hence the patterns of in situ produced chlorophyll through submesoscale vertical nutrient injection.

Citation: Lehahn, Y., F. d'Ovidio, M. Lévy, and E. Heifetz (2007), Stirring of the northeast Atlantic spring bloom: A Lagrangian analysis based on multisatellite data, J. Geophys. Res., 112, C08005, doi:10.1029/2006JC003927.

\section{Introduction}

[2] The North Atlantic spring bloom is a pronounced increase in phytoplankton biomass, triggered by the shallowing of the mixed layer (ML) below Sverdrup's critical depth [Sverdrup, 1953]. As a result of the northward propagation of the water column restratification, the bloom propagates northward throughout spring. This propagation is followed by a northward propagation of the large-scale surface chlorophyll front between the productive waters in the north and the oligotrophic waters in the south [Lévy et al., 2005b].

[3] The North Atlantic spring bloom is a basin-scale phenomenon [Esaias et al., 1986; Follows and Dutkiewicz, 2002] with great importance for ocean productivity and has been the focus of several field experiments, including the JGOFS North Atlantic Bloom Experiment (NABE) and the Programme Océan Multidisciplinaire Méso Echelle (POMME). In situ observations made in the framework of these projects have shown that even though the bloom is a large-scale phenomenon, mesoscale variability in the bio-

\footnotetext{
${ }^{1}$ Laboratoire d'Océanographie et du Climat: Expérimentations et Approches Numériques, Institut Pierre-Simon Laplace, Centre National de la Recherche Scientifique, Paris, France.

${ }^{2}$ Department of Geophysics and Planetary Sciences, Tel Aviv University, Tel Aviv, Israel.

${ }^{3}$ UMR 8539, Laboratoire de Météorologie Dynamique, Ecole Normale Supérieure, Paris, France.

Copyright 2007 by the American Geophysical Union. 0148-0227/07/2006JC003927
}

logical fields is also observed [Robinson et al., 1993; Mémery et al., 2005].

[4] The aim of this paper is to elucidate some of the mechanisms by which the geostrophic velocity field acts on the pattern formation of mesoscale and submesoscale chlorophyll patches. This is done by focusing on the spring bloom in the northeast Atlantic and analyzing altimetry data (TOPEX/POSEIDON, ERS, Jason and Envisat), sea color images from the Sea-viewing Wide Field-of-view Sensor (SeaWiFS) and when relevant sea surface temperature (SST) images from the Advanced Very High Resolution Radiometer (AVHRR).

[5] The analysis is based on the derivation of Lagrangian diagnostics from the time-dependent geostrophic velocity field, and more precisely transport barriers and trajectories of synthetic passive tracers. As we shall see, these Lagrangian tools are very effective in reconstructing the specific effect of horizontal stirring on individual patterns. They provide a complementary approach to previous techniques derived from turbulence, like calculation of turbulent diffusion and spectral analysis, that are instead very powerful in detecting statistical properties (see for instance Pasquero [2005] and the review by Martin [2003]). Lagrangian techniques are based, in general, on the identification of the velocity field characteristics along particle trajectories. They are very well suited for diagnosing properties of tracers like chlorophyll, since they allow to quantify the dynamical properties experienced by a parcel of water during its motion. In spite of the fact that Lagrangian tools are extensively employed for atmospheric tracers, their use for chlorophyll has been confined to a small number of 
isolated cases. Abraham et al. [2000] studied a local phytoplankton bloom initiated by the iron fertilization of a patch of water in the Southern Ocean and highlighted the efficiency of the deformation field associated with mesoscale circulation to produce long and thin horizontal chlorophyll filaments in a short period; Toner et al. [2003] used a Lagrangian method to describe the evolution of two chlorophyll plums in the Gulf of Mexico.

[6] In this work we present a systematic analysis of the impact of horizontal stirring in modulating chlorophyll variability, covering the period of the spring bloom in six consecutive years (1998-2003). The main Lagrangian concept that we use is the notion of unstable manifold. Unstable manifolds are material lines with a converging, transverse dynamics. Owing to the converging dynamics transverse to them, unstable manifolds act on any advected tracer as "sticking" transport barriers, creating fronts and filaments. The concept of unstable manifold suggests some typical scenarios induced by the advection of postbloom chlorophyll and by in situ phytoplankton production. The suggested scenarios are verified by comparing chlorophyll fronts and filaments with reconstructed unstable manifolds. In order to isolate the effect of the 2D transport, chlorophyll patterns are compared with distribution patterns of synthetic passive particles that are advected by the satellite derived geostrophic velocities. When relevant, SST structures are also being compared.

[7] The good agreement between the expected and the observed chlorophyll patterns indicates the central role of horizontal stirring in shaping the spatial phytoplankton variability in the northeast Atlantic for postbloom and locally produced chlorophyll. Remarkably, the unstable manifolds allow to predict some structures that are below the altimetric resolution. These small-scale patterns result by the time-dependent (chaotic) evolution of mesoscale coherent structures of the velocity field and can be captured by the Lagrangian detection of transport barriers.

[8] The paper is organized as follows. Section 2 gives an overview of the regional context. Section 3 details the data and methods, with an emphasis on the concept of unstable manifolds and their interaction with postbloom chlorophyll and in situ production. In section 4 we compare chlorophyll patterns and passive tracer evolution with unstable manifolds, focusing on three typical structures. The results are discussed and concluded in section 5.

\section{Regional Context}

[9] The study is focused on a $20^{\circ}$ latitudinal band in the northeast Atlantic $\left(13^{\circ} \mathrm{W}-23^{\circ} \mathrm{W} / 30^{\circ} \mathrm{N}-50^{\circ} \mathrm{N}\right.$, Figure 1a). This area overlaps the region of the POMME experiment that was conducted in order to study the role of mesoscale eddies in the formation and subduction of $11^{\circ}$ and $13^{\circ}$ mode waters in the northeast Atlantic. POMME included a series of interdisciplinary field experiments over a seasonal cycle from October 2000 to October 2001, with more intense observations during the period of the spring bloom [Mémery et al., 2005].

[10] This domain covers part of the subpolar gyre and part of the subtropical gyre. It is characterized by a sharp transition between an area of deep winter mixed layer depth (MLD) in the north and an area of shallower MLD in the south [Arhan et al., 1994; Paillet and Arhan, 1996a]. The strong meridional gradient in winter MLD is clearly seen in the recent climatology of de Boyer-Montegut et al. [2004], with values reaching $220 \mathrm{~m}$ in the north and $100 \mathrm{~m}$ in the south. Although the area is of relatively low eddy kinetic energy (EKE) [Stammer et al., 2006], the large-scale circulation is dominated by cyclonic and anticyclonic mesoscale eddies [Assenbaum and Reverdin, 2005; Le Cann et al., 2005], with a typical eddy size of one to two hundreds of kilometers (Figure 2a). The mean Ekman transport is directed southward. The combination of the winter meridional MLD gradient with the mean southward circulation leads to subduction of subpolar mode water [Paillet and Arhan, 1996a, 1996b].

[11] In terms of chlorophyll, the area is characterized by a strong meridional gradient and relatively week zonal variations (Figure 1a). The north is productive with high values of chlorophyll (annual mean $>0.4 \mathrm{mg} / \mathrm{m}^{3}$ ), and the south is oligotrophic with low chlorophyll values (annual mean $<0.1 \mathrm{mg} / \mathrm{m}^{3}$ ). The large-scale meridional chlorophyll gradient results essentially from the meridional variations in winter convective supply of nutrients. In the north, deeper winter ML combined with a shallower nutricline allows higher supply of nutrients to the euphotic layer. In the south, the winter source of nutrients is much smaller and the region is oligotrophic [Williams et al., 2000; Williams and Follows, 2003].

[12] Chlorophyll seasonality is mainly driven by variations in the MLD. The latter result from changes in the solar flux and atmospheric forcings. The study is focused on the period of the subpolar spring bloom (crudely between the months of March and July), when the meridional chlorophyll gradient is the strongest (Figure 1b) and the water column is stratified.

\section{Data and Method}

\subsection{Satellite Data}

[13] Daily images of chlorophyll concentrations were processed from high-resolution $(1 \mathrm{~km})$ Level 2 SeaWiFS data, obtained from the NASA Goddard Distribution Active Archive Center (DAAC). Data were processed using NASA's SeaWiFS Data Analysis System (SeaDAS software package). Over 700 chlorophyll images during the spring bloom and for the period 1998-2003 were examined. We focused on images overlapping the large-scale meridional chlorophyll gradient (see Figure $1 \mathrm{~b}$ ). We selected an image size of $7^{\circ} \times 7^{\circ}$ in order to encompass several eddies. Because of high cloud cover, only 23 such images could be exploited (Table 1). In these images, the cloud coverage is smaller than $30 \%$.

[14] Sea-level anomalies (SLA) were obtained from the AVISO database (http://www-aviso.cnes.fr). The distributed global product combines altimetric data from the TOPEX/ POSEIDON and ERS missions, from Jason-1 for data after December 2001 and from Envisat for data after March 2002. The SLA is gridded on a $1 / 3^{\circ} \times 1 / 3^{\circ}$ Mercator grid, with one data file every seven days. The Rio-05 mean dynamic topography (MDT) [Rio and Hernández, 2004] was added to the SLA to get the sea surface height (SSH). Geostrophic velocities were calculated from this $\mathrm{SSH}$, by solving the equation for geostrophic equilibrium [Pedlosky, 1987] with centered, finite differences. 
a) SeaWiFS chlorophyll (1998-2003 mean)

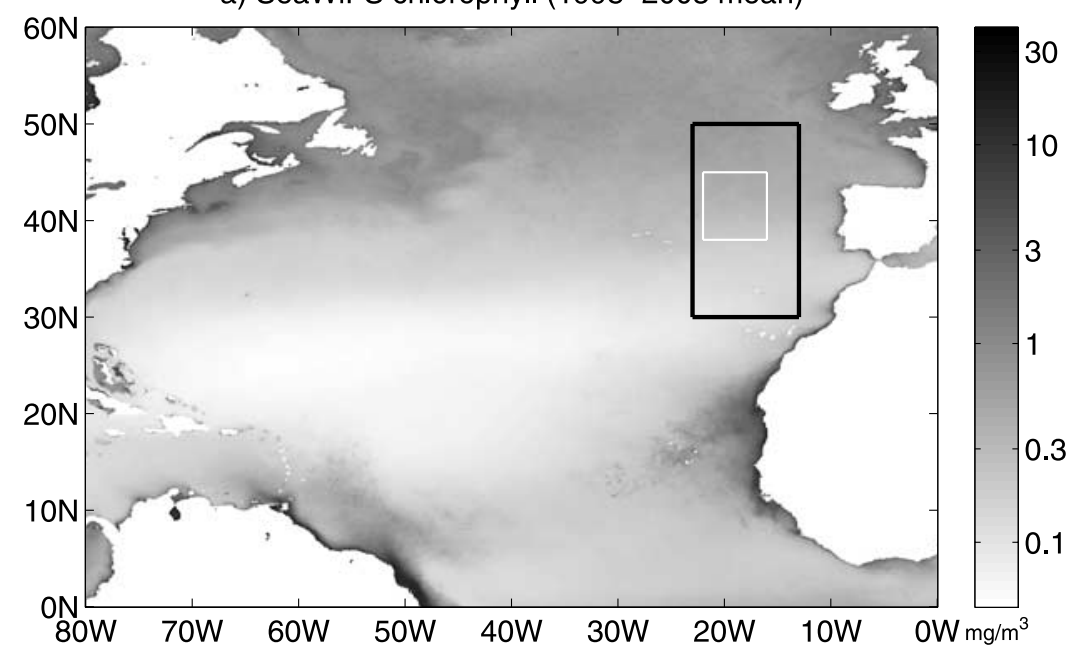

b) SeaWiFS chlorophyll (zonal averages, 13-23W)

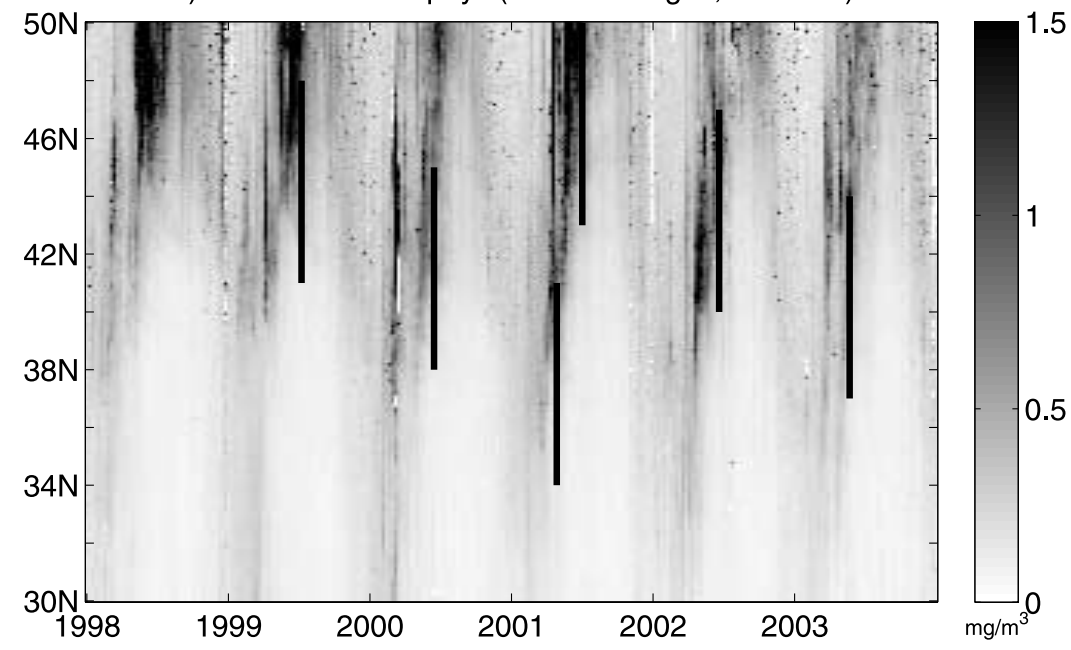

Figure 1. (a) Average SeaWiFS chlorophyll concentrations in the North Atlantic during the period 1998-2003. The black frame marks the location of the region examined in this study. The white frame marks the location of the POMME experiment. (b) Time series of zonally averaged $\left(13^{\circ}-23^{\circ} \mathrm{W}\right)$ chlorophyll. The six vertical black lines mark the time and meridional extension of the six images shown in Figure 7.

[15] Daily AVHRR data with a resolution of $1 \mathrm{~km}$ were processed toward SST images by Meteo France in Lannion. The processing only covers the period of the POMME experiment (from October 2000 to October 2001).

\subsection{Lagrangian Trajectories and Finite-Size Lyapunov Exponent Calculation}

[16] The trajectories used for the passive tracer experiments and for the Lyapunov exponent calculation have been computed by integrating the geostrophic velocities with a Runge-Kutta scheme of the fourth order with a fixed time step of 6 hours. The velocities derived from the altimetric data has been interpolated in space and time with a multilinear algorithm. Transport properties are studied by computing Lyapunov exponent on the sea surface [Abraham and Bowen, 2002; d'Ovidio et al., 2004; Waugh et al., 2006].
More precisely we use the finite-size method and parameters as in work by d'Ovidio et al. [2004]. Lyapunov exponents are obtained by measuring the exponential divergence of nearby trajectories. For the final deformation we choose $0.8^{\circ}$ while for the initial separation we used a value of $0.01^{\circ}$, that also defines the resolution of the Lyapunov map. By equating the initial separation to the resolution of the map, we guarantee that all the space is sampled at least once, and no more than once. In order to avoid the dependence on the orientation of the pairs, Lyapunov exponents are obtained by diagonalizing the linear transformation of a square whose diagonals are formed by two pairs [Ott, 1993]. As we shall see in the next section, in this paper we are interested in unstable manifolds of hyperbolic points (see Appendix A for a detailed discussion of the method). Such structures can be identified as local maxima (ridges) of 

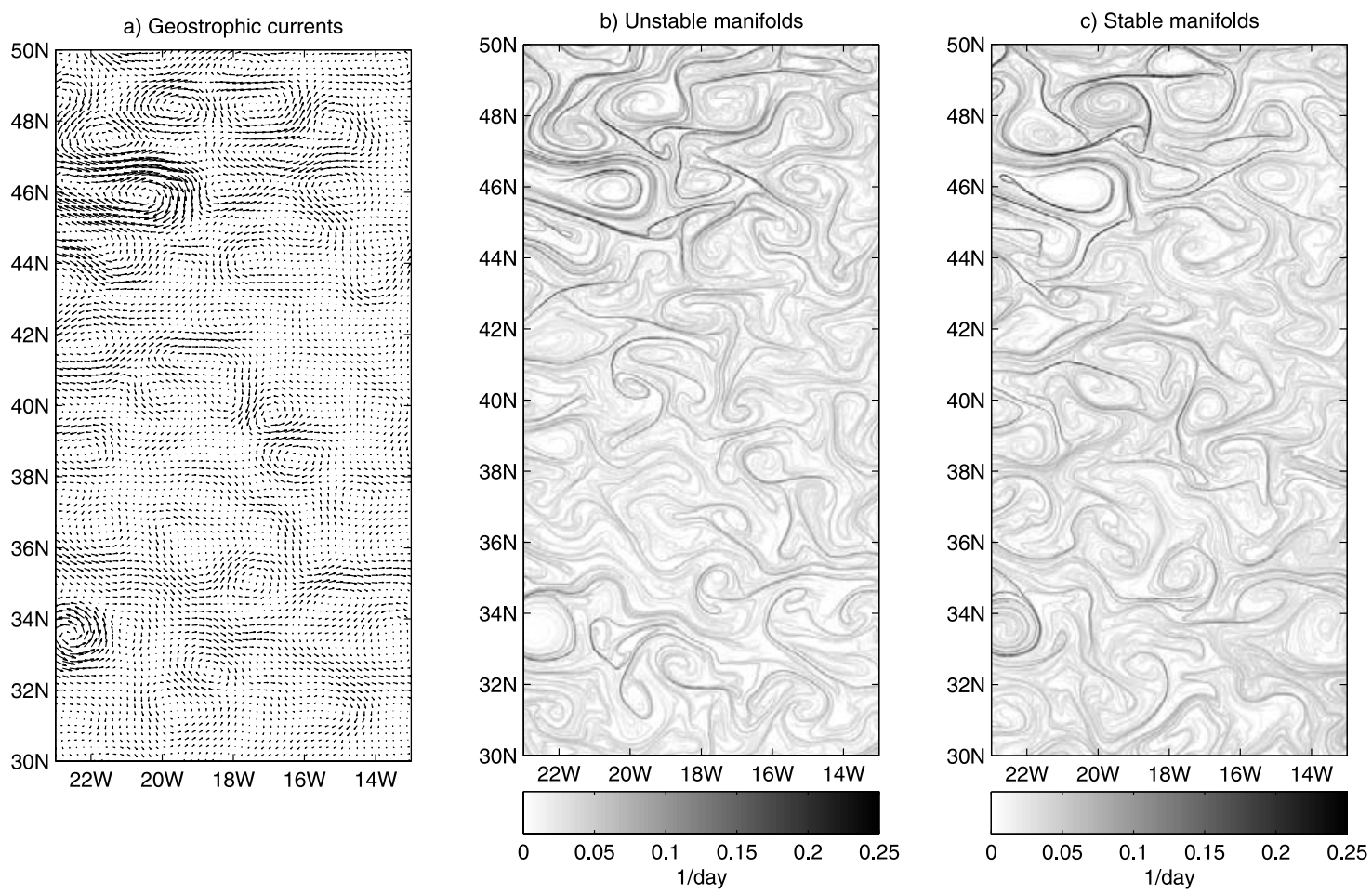

Figure 2. (a) Geostrophic velocity field, (b) stable manifolds, and (c) unstable manifolds for 20 July 1998.

Finite-Size Lyapunov Exponents (FSLE) values (see Appendix A). The intensity of the Lyapunov exponent measures the timescale at which a tracer relaxes over the manifold, i.e., the intensity of the convergent field. The strong manifolds are therefore the manifolds that most affect tracers.

\subsection{Lagrangian Analysis of Transport Barriers}

[17] The transport properties of the currents are described in terms of hyperbolic points and manifolds embedded in the velocity field. For the reader not familiar with these concepts, we now provide some brief overview, and refer to more specific works [Haller and Yuan, 2000; Boffetta et al., 2001; Koh and Legras, 2002; Mancho et al., 2004; Wiggins, 2005].

[18] Let us start by a region dominated by mesoscale eddies, like the one depicted in Figure 3a, and suppose that the velocity field is stationary. Without calculation, one can detect a point at the intersection of a diverging and converging region (black dot). Such hyperbolic points are of special relevance for transport, as shown in Figure 3b. A passive tracer initialized nearby the hyperbolic point is affected at the same time by contraction along the converging direction (called stable manifold of the hyperbolic point) and stretching along the diverging direction (unstable manifold). The tracer approaches the hyperbolic point as a thinner and thinner filament and eventually aligns along the unstable manifold. Note that owing to the converging transverse component of the velocity field, the tracer cannot cross such a line. For this reason, unstable manifolds of hyperbolic points act as transport barriers and control the formation of fronts. An example of this is shown in Figure $4 \mathrm{a}$ where two patches initialized in the vicinity of the hyperbolic point are advected backward (blue) and forward (green) in time and approach respectively the stable and unstable manifold as thin filaments after a few days.

[19] The above description of hyperbolic structures is rigorous for stationary velocity fields, but can still be applied to the time-dependent case, provided that the evolution of the velocity field is on a slower timescale than

Table 1. Days and Coordinates of the Images Analyzed in This Study

\begin{tabular}{lcc}
\hline \multicolumn{1}{c}{ Date } & Longitude, ${ }^{\circ} \mathrm{W}$ & Latitude, ${ }^{\circ} \mathrm{N}$ \\
\hline 23 July 1998 & $13-20$ & $42-49$ \\
7 April 1999 & $13-20$ & $37-44$ \\
22 April 1999 & $13-20$ & $37-44$ \\
16 May 1999 & $16-23$ & $41-48$ \\
17 May 1999 & $13-20$ & $37-44$ \\
22 May 1999 & $16-23$ & $41-48$ \\
16 June 1999 & $16-23$ & $41-48$ \\
7 July 1999 & $16-23$ & $41-48$ \\
15 May 2000 & $13-20$ & $38-45$ \\
13 June 2000 & $16-23$ & $38-45$ \\
1 April 2001 & $15-22$ & $34-41$ \\
9 April 2001 & $15-22$ & $34-41$ \\
15 April 2001 & $15-22$ & $34-41$ \\
25 April 2001 & $15-22$ & $34-41$ \\
27 May 2001 & $13-20$ & $40-47$ \\
19 June 2001 & $13-20$ & $40-47$ \\
22 June 2001 & $16-23$ & $41-48$ \\
30 June 2001 & $14-21$ & $43-50$ \\
18 June 2002 & $16-23$ & $40-47$ \\
1 July 2002 & $15-22$ & $43-50$ \\
21 June 2003 & $13-20$ & $37-44$ \\
14 June 2003 & $16-23$ & $42-49$ \\
23 June 2003 & $16-23$ & $42-49$ \\
\hline
\end{tabular}


a

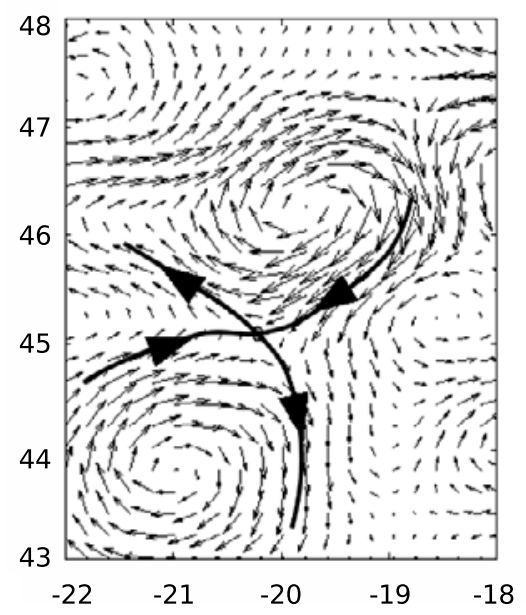

b

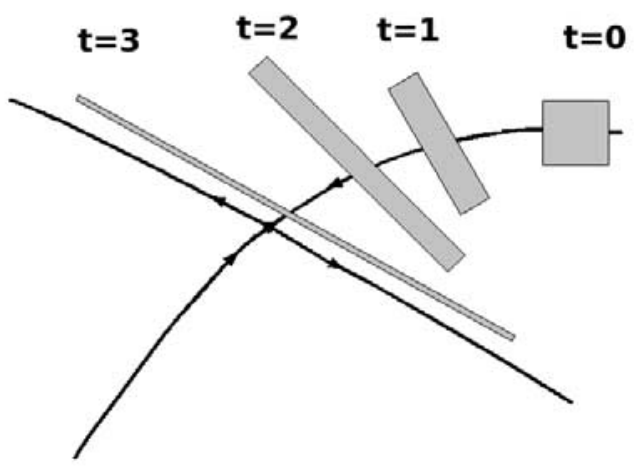

Figure 3. (a) Geostrophic velocity field (small arrows) and pathways (thick arrows) of convergence and divergence toward and from a hyperbolic point. (b) An illustration of the stretching of a passive tracer that is initialized near a hyperbolic point.

tracer advection. If this is the case, a tracer can relax over a manifold (the timescale being the inverse of the value of the Lyapunov exponent, as explained later), and then evolve together with the manifold. For the ocean, there is a clear timescale separation, because the propagation of the eddies (and thus of the unstable manifolds) is much slower than the mesoscale velocity field. Midlatitude mesoscale eddies have typical lifetimes of several months and a speed of the order of $10 \mathrm{~km} /$ week while mesoscale velocities are 1 order of magnitude larger. While a tracer cannot cross an unstable manifold, it is forced by the transverse, converging velocity to stick to it. As the manifold moves, the tracer front is thus shaped and transported. In particular, in the case of a timedependent field, unstable moving manifolds evolve in convoluted and lobular structures that allow a tracer to intrude other regions. Therefore moving unstable manifold does not only act as transport barriers, but also control exchange and mixing. Figures $4 \mathrm{c}-4 \mathrm{f}$ compares the cases of stationary (i.e., frozen in time) and time-dependent velocities. In the first case, synthetic tracer trajectories are forced to follow altimetric isolines. Indeed, in geostrophic balance, altimetric isolines are the streamlines and therefore for a stationary velocity field the altimetric isolines coincide with the trajectories. A consequence of this stationarity is that the eddies are characterized by concentric closed circles ("tori", as they are properly referred to) that perfectly isolate from the surrounding (Figures $4 \mathrm{c}$ and $4 \mathrm{e}$ ). On the other hand, for the case of time-dependent velocities, the identity between trajectories and streamlines does not hold anymore. In particular, a tracer released inside an eddy does not follow a closed path and after one revolution does not come back exactly to its initial position. In this way, the impermeable barriers formed by the concentric isolines are replaced by spirals, as can be easily seen by detecting transport barriers with the Lyapunov exponent calculation (Figure 4f), or simply by releasing a tracer (Figure 4d). The spirals are tighter and closely resemble impermeable tori where the streamlines are not strongly affected by the time dependency, that is, at the eddy cores. In these regions, passive tracers are trapped for a long time and can escape the eddy only after several revolutions. The picture of an isolated eddy core is indeed in agreement with observed trajectories of released floaters in the POMME region [ $L e$ Cann et al., 2005] and, as we shall see, has a strong effect on the shaping of submesoscale chlorophyll patterns. On the other hand, tori break in looser spirals at the periphery, allowing a relatively stronger exchange. The formation of spirals is a purely dynamical phenomenon, that is controlled by the time variability of the velocity field and not by the spatial scale of the velocity field. For this reason, a passively advected tracer can be distributed with spatial structures that are below the resolution of the velocity field itself. Note however, that spirals can also result from nongeostrophic stationary velocity fields, such as the wellknown Ekman spiral.

[20] Probing a velocity field by advecting a passive tracer as in Figure 4a is a way of detecting unstable manifolds. The finite-size Lyapunov exponent technique [Aurell et al., 1997; d'Ovidio et al., 2004] that we use in this work is a systematic way of doing the tracer experiment of Figure 4a. By paving the domain and repeating this process, we measure the dynamical (Lagrangian) stretching rates, that is known to provide good candidates for points along unstable manifolds. The global result is the full complexity of these manifolds (Figures $2 \mathrm{~b}$ and $2 \mathrm{c}$ ). For clarity, we define the strong manifolds as the manifolds associated with the largest 10\% FSLE values (Figure 4b).

\subsection{Geostrophic Transport Barriers and Chlorophyll Patterns}

[21] The main idea of the previous section that will guide us in the analysis of geostrophic velocity field and chlorophyll images, is that unstable manifolds can be regarded as "sticking" transport barriers, that move and shape the fronts of any advected tracer. The use of Lagrangian diagnostics for the characterization of tracer distributions is extensively made for the atmosphere [see, e.g., Koh and Legras, 2002, and references therein], and much less for the ocean. For the 
a) Particles (initial \& final)

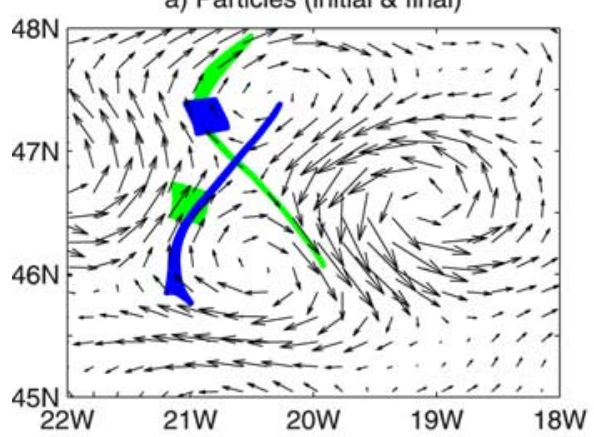

c) Particles(stationary velocity field)

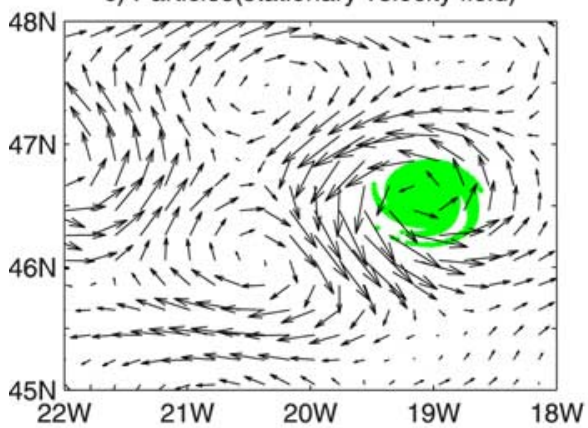

e) Unstable manifolds(stationary velocity field)

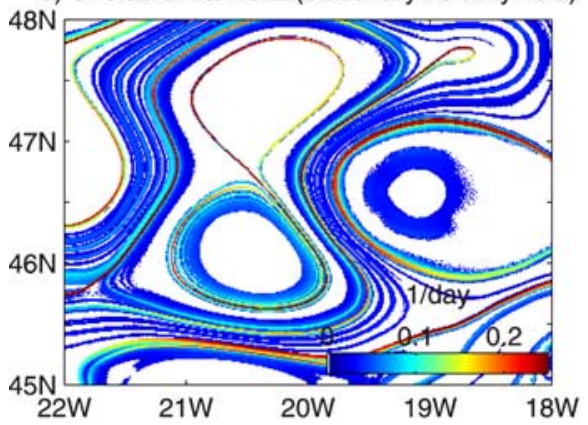

b) Strong stable(b) \& unstable (g) manifolds

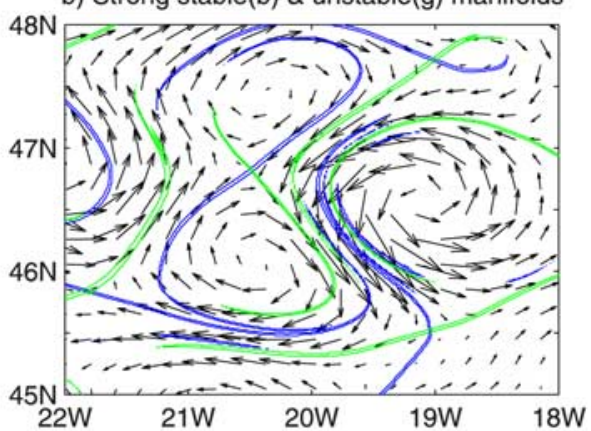

d) Particles(time-dependent velocity field)

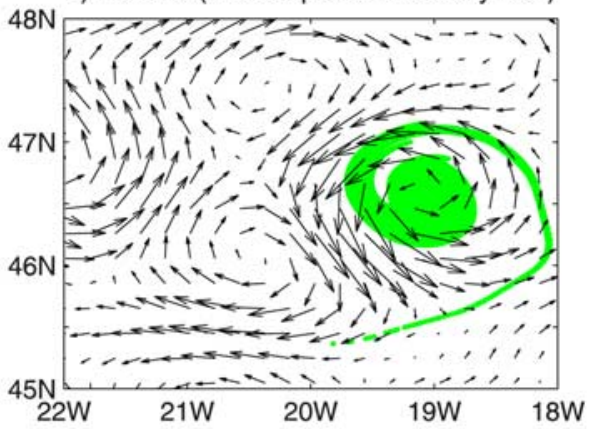

f) Unstable manifolds(time-dependent velocity field)

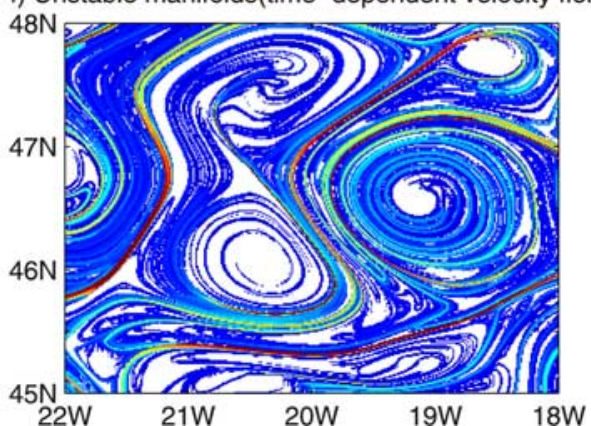

Figure 4. Superposition of an Eulerian geostrophic velocity field with (a) two snapshots (15 days apart) of Lagrangian particles advected forward and backward (respectively, green and blue dots). (b) Strong unstable and stable manifolds (respectively, green and blue lines). (c) Lagrangian particles advected by a stationary geostrophic velocity field, and (d) Lagrangian particles advected by a time-dependent geostrophic velocity field. (e) Unstable manifolds calculated from a stationary geostrophic velocity field, and (f) unstable manifolds calculated from a time-dependent geostrophic velocity field.

case of the northeast Atlantic and phytoplankton patterns, we will focus on two mechanisms that point on a strong spatial correlation between unstable manifolds and chlorophyll fronts. The first one is a direct effect of transport on postbloom chlorophyll. The second one is an indirect effect of the horizontal hyperbolic regions to support local production of chlorophyll, mediated by vertical advection of nutrients upon unstable manifolds. Let us now elaborate on the two mechanisms and speculate over the features that we expect to find in the satellite observations. The North Atlantic spring bloom is primarily induced by a shallowing of the mixed layer, and can be seen as a large-scale, northward propagating chlorophyll front. Over a short period (1-2 weeks) a large reservoir of phytoplankton is created. This chlorophyll pool is affected by the horizontal transport. Chlorophyll relaxes onto the unstable manifolds and is then trapped and advected by lobes, and ultimately mixed with poorer water. Recalling the discussion of section 3.2, we therefore expect three types of patterns as the direct effect of stirring of the postbloom chlorophyll pool: (1) lobular fronts in correspondence of strong manifolds; (2) submesoscale patterns inside eddies, characterized by weakly permeable cores (chlorophyll spots) connected to the periphery by spiraling lobes of unstable manifolds; and (3) filaments aligned to the diverging direction for chlorophyll initialized over hyperbolic points. Figures $5 \mathrm{a}-5 \mathrm{c}$ presents in a schematic view the genesis of these three patterns.

[22] In spite of the fact that the geostrophic velocities do not contain vertical component, a relationship between unstable manifolds and in situ chlorophyll production through upwelling of nutrients might be expected [Lapeyre and Klein, 2006]. The vertical velocity field in the POMME 


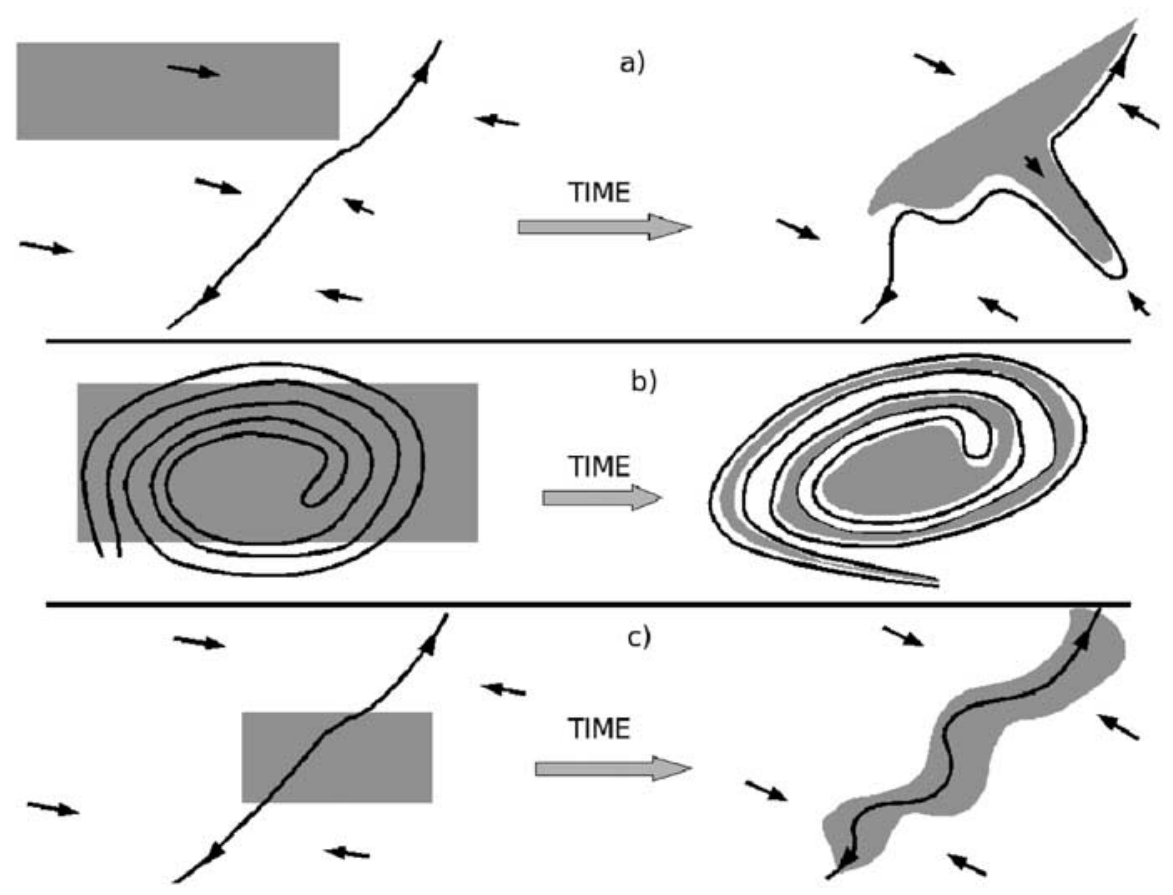

Figure 5. Postbloom chlorophyll pattern formation by horizontal advection. (a) Front. A patch of phytoplankton is conveyed toward the unstable manifold by the converging field and then sticks to it. The unstable manifold typically folds in lobes and meanders leading to intrusion filaments of chlorophyll. Such lobes are due to the time dependence of the geostrophic velocity field and may move against the direction of the geostrophic velocities. In fact, the whole geostrophic velocity field moves. (b) Spiral. If the phytoplankton patch is formed over an eddy, chlorophyll is advected outward by a spiraling unstable manifold and chlorophyll-poor water intrudes on a complementary lobe. The chlorophyll spiral unwinds outward according to the motion of the unstable manifold, which may be different from the rotation of the eddy (see for instance Figure 4d). (c) Filament. If the phytoplankton patch is formed over the unstable manifold, it is elongated along in a filament over the unstable manifold due to the converging field.

region was described by Legal et al. [2006] on the basis of SEASOR data combined with the analysis of altimetry data. Their analysis revealed a vertical velocity field $w$, involving elongated thin structures, in agreement with previous estimations from numerical simulations in the POMME region [Paci et al., 2005]. The 3D dynamics involved is the restoration of the thermal wind balance within small-scale density filaments that are elongated by horizontal stirring processes [Hakim et al., 2002]. It induces a strong correlation between density anomalies and $w$, with $w$ positive in lighter filaments and negative in denser filaments within regions of high strain. In Figure 6 we apply this mechanism to an SST front shaped by an unstable manifold and sketch the expected effect on plankton formation. Note that the phytoplankton is not expected to be directly in phase with the upwelling cell because of the strong ageostrophic transport toward the downwelling cell. Instead, it is expected to be shifted toward the unstable manifold. This mechanism acts in phase with the previous one (Figures $5 \mathrm{a}-5 \mathrm{c}$ ), producing chlorophyll filaments along unstable manifolds.

\section{Results}

\subsection{Chlorophyll Fronts}

[23] Figure 7 shows six examples of SeaWiFS chlorophyll images overlaid with the geostrophic velocities (white

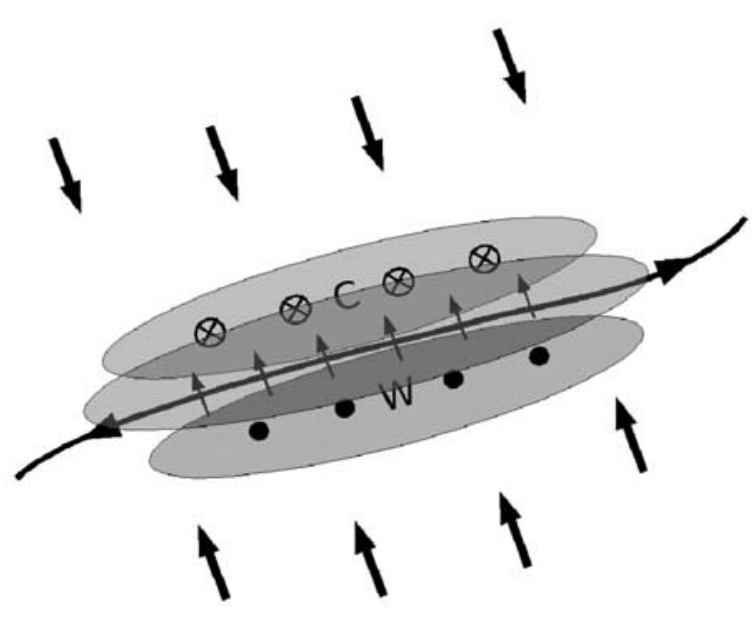

Figure 6. Chlorophyll pattern formation by local nutrient upwelling. Upwelling and downwelling occur respectively on the negative (warm) and positive (cold) anomaly. By continuity, an ageostrophic circulation connects the warm branch to the cold branch [Hakim et al., 2002; Legal et al., 2006; Lapeyre and Klein, 2006]. Nutrients are advected to the photic layer along the warm branch and then conveyed toward the cold branch by the ageostrophic circulation. This generates a local bloom on the top of the unstable manifold. 
a) July 7, 1999

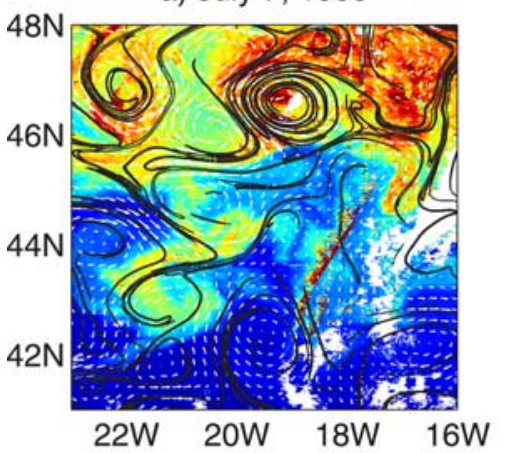

c) April 24, 2001

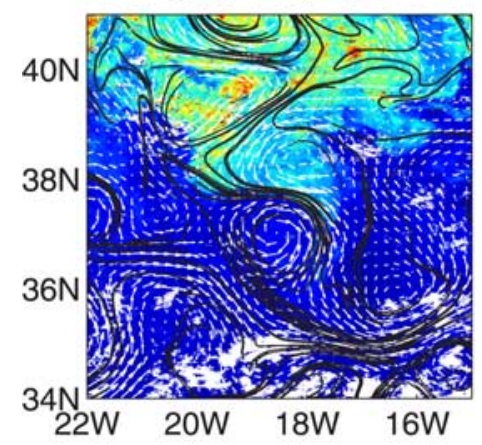

e) June 18, 2002

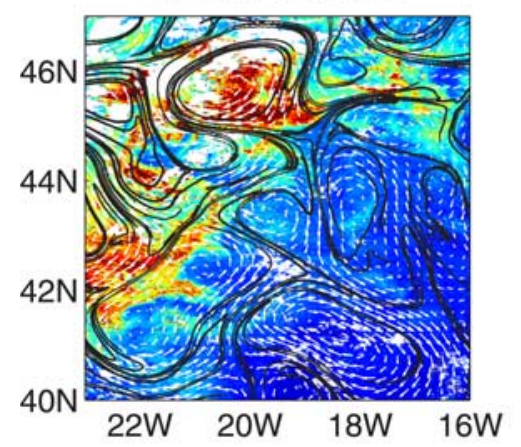

b) June 13,2000

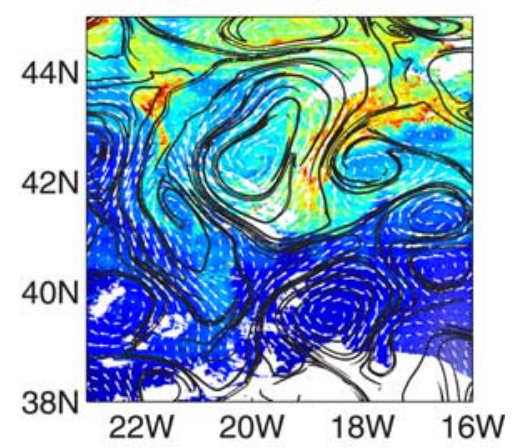

d) June 30, 2001

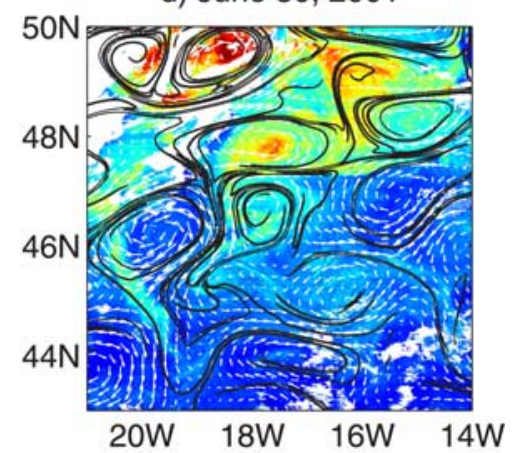

f) May 21, 2003

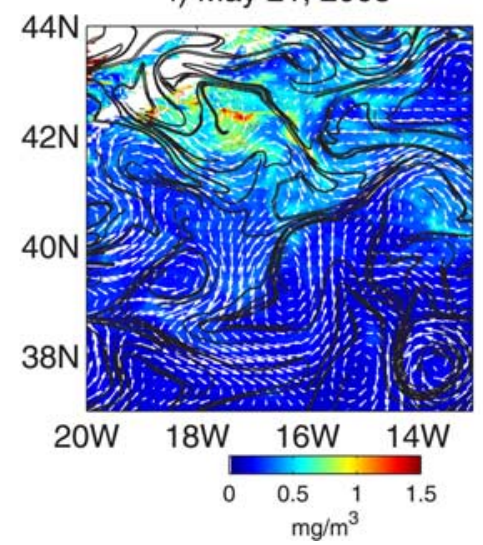

Figure 7. Superposition of strong unstable manifolds (black lines) and of the geostrophic velocities (white arrows) over SeaWiFS chlorophyll concentrations (colors). The timing and meridional extension of the images with respect to the large-scale chlorophyll variability are marked by black lines in Figure 1b. Throughout the paper, chlorophyll images are plotted using the same colorscales as in Figure $7 \mathrm{f}$.

arrows), and with the unstable manifolds (black lines). In order to facilitate the comparison between the fields, only the strong manifolds are plotted. The time-space location of these six images with respect to the evolution and northward propagation of the bloom is indicated by the black lines on Figure 1b. Note that all images cover the large-scale chlorophyll front associated with the bloom propagation. As mentioned before, this large-scale chlorophyll front mainly results from the larger winter convective supply of nutrients in the north. Figure 7 reveals that the large-scale chlorophyll front is shaped by the mesoscale flow field. Furthermore, there are indications of intrusions, associated as well with the flow field, of rich water to the south and of poor water to the north (see for instance the patch of relatively rich water at around $21^{\circ} \mathrm{W} / 43^{\circ} \mathrm{N}$ in Figure $7 \mathrm{a}$, to which we will come back in section 4.2).

[24] The relationship between the chlorophyll distribution and the flow field is evidenced by the alignment of the chlorophyll front (and notably of chlorophyll intrusions) with the unstable manifolds and supports the scenario described in Figure 5a. Note that this relationship is much less distinct when using the instantaneous velocity field instead of the manifolds. Similarly, the orientation of the intrusion filaments (of either chlorophyll-rich or poor water) is in much better agreement with the unstable manifolds than with the instantaneous velocity field. This seemingly 


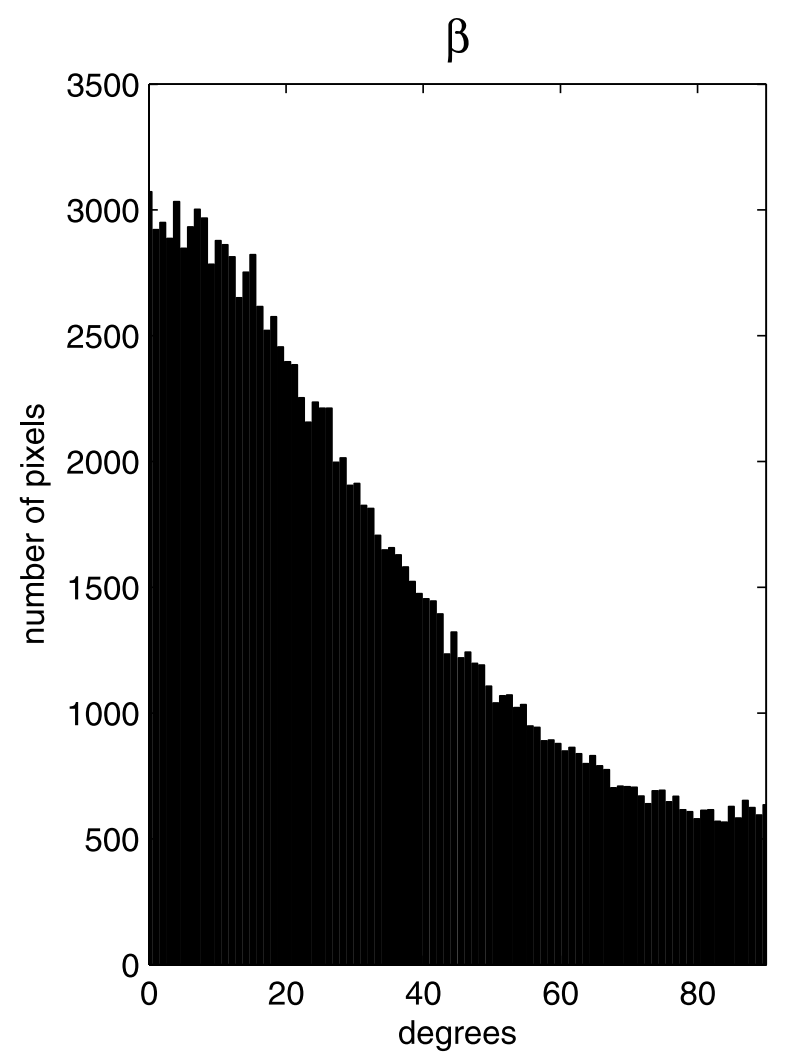

Figure 8. Distribution of the angle $\beta$, between the direction of the chlorophyll isolines and the direction of the unstable manifolds. Only pixels with strong manifolds and strong chlorophyll gradients are accounted for. A perfect agreement corresponds to $\beta=0^{\circ}$ (see text).

counterintuitive observation comes from the fact that individual water parcels follow the velocity field, while fronts are shaped by the time evolution of the velocity field, as depicted in Figure 5a. The overall agreement between the manifolds and the chlorophyll distribution is quantified by the histogram in Figure 8. This histogram describes the distribution of the angle $\beta$ between the direction of the unstable manifold and of the chlorophyll isolines, so that a perfect agreement corresponds to $\beta=0^{\circ}$ and an absolute mismatch corresponds to $\beta=90^{\circ}$. In order to filter out the highest frequencies (that are not detected by the manifolds, see further in the discussion), the chlorophyll images are first smoothed with a running window of $30 \mathrm{~km}$. For the overall 23 images, all pixels including both strong manifolds and $10 \%$ of the strongest values of chlorophyll gradient are examined. The angle $\beta$ is smaller than $20^{\circ}$ in almost half (43\%) of the pixels and is larger than $70^{\circ}$ in only $9 \%$ of them.

[25] This alignment of the chlorophyll front along unstable manifolds confirms that the meandering of this front primarily results from transport barriers stemming from the geostrophic advection. In Figure 9, two snapshots of the same chlorophyll front 15 days apart (corresponding to the front around $19^{\circ} \mathrm{W} / 38^{\circ} \mathrm{N}$ in Figure 7c) provide an opportunity for exploring the time evolution of the chlorophyll front versus the evolution of the unstable manifolds. Figures 9a and 9b shows that, during the 15 days, the chlorophyll front and the unstable manifolds have both moves toward the south. A similar coherency is found with the SST front (Figures 9c and 9d). Incidentally, one can also note that smaller-scale structures can be seen along the fronts in the chlorophyll and SST images and are not captured by the manifolds.

[26] In order to further examine the role of 2D geostrophic advection in shaping the chlorophyll front, we eliminate other processes (such as phytoplankton growth and decay, and vertical advection) by simulating the evolution of synthetic, purely passive particles. These passive particles are initially organized as a large-scale meridional front (not shown). This front resembles crudely the chlorophyll distribution at the large scale (see Figure 1b) in the initial date of the simulation (26 March 2001). The passive particles are solely advected by the horizontal geostrophic velocities, derived from the satellite altimetry data. The evolution of the particles (Figures 9e and 9f) shows a clear correspondence with the fronts of chlorophyll and SST at the mesoscale.

\subsection{Chlorophyll Spirals}

[27] Figure 7 shows several examples of cyclonic and anticyclonic eddies with chlorophyll levels higher than their surroundings: two cyclones on Figure 7 a located at $21^{\circ} \mathrm{W} /$ $43^{\circ} \mathrm{N}$ and $22^{\circ} \mathrm{W} / 47^{\circ} \mathrm{N}$, one anticyclone on Figure $7 \mathrm{~d}$ at $18^{\circ} \mathrm{W} / 47.5^{\circ} \mathrm{N}$, and another anticyclone on Figure $7 \mathrm{e}$ at $20.5^{\circ} \mathrm{W} / 45.5^{\circ} \mathrm{N}$. The chlorophyll distribution within these eddies is not homogeneous. It often has a spiral like shape, and occasionally a spot at the center, particularly remarkable in Figure $7 d$.

[28] As depicted in Figure 5b, the time-dependency of the velocity field is enough to generate inward and outward flows toward and from geostrophic eddies, which have the shape of spirals. This spiral flow can either introduce relatively rich waters into the eddy, thus creating a positive anomaly within the eddy, or vice versa.

[29] In order to test whether this mechanism of horizontal enrichment of eddies could apply to the positive anomaly located at $21^{\circ} \mathrm{W} / 43^{\circ} \mathrm{N}$ in Figure $7 \mathrm{a}$, Figure 10 zooms over this eddy and shows the evolution of synthetic, passive particles initially organized as a front north of the eddy (Figure 10a). This integration shows that, within the timescale of a month, the particles are entrained within the eddy in a spiral manner (Figure 10c) that resembles the chlorophyll spiral seen in the data (Figure 10d). Note that the shape of the spiral is also controlled by the location of the unstable manifolds (Figure $7 \mathrm{a}$ and 10c).

[30] This example highlights how a large-scale front of chlorophyll can cascade to smaller scales by filamentation into spirals that eventually enrich eddies. In this particular example, the eddy is located to the south of the large-scale chlorophyll front and the unstable manifolds associated with the eddy are crossing it. This allows cross-front exchange and penetration of chlorophyll to the south of the front.

[31] Small spots at the core of some eddies (either as a positive anomaly as in Figure $7 \mathrm{~d}$ or as a negative anomaly as in Figure 7a) are shielded against mixing with the exterior by the spiral flow. Indeed, the discrepancy between trajectories and streamlines in the case of a nonstationary velocity field is more pronounced at the eddy periphery than at its core, since the inner core is less affected by the time- 
a) Chlorophyll, April 92001

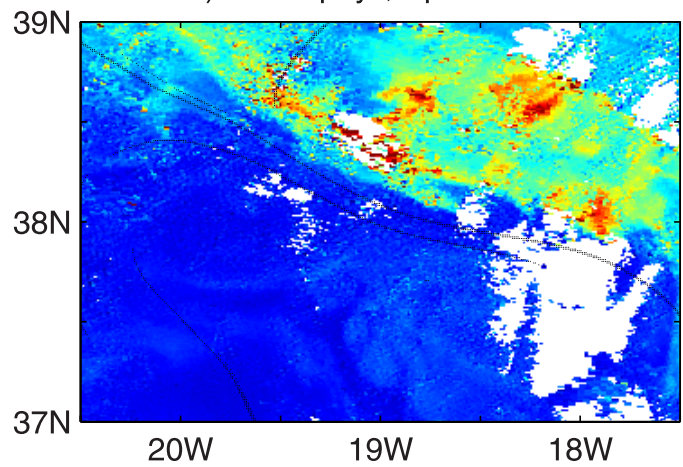

c) SST, April 132001

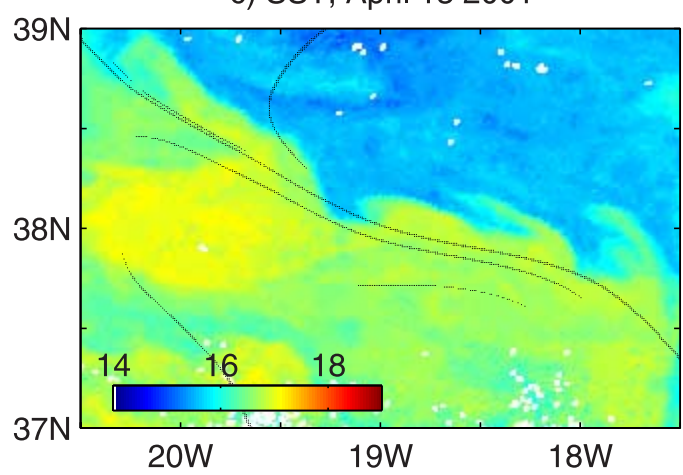

e) Particles, April 92001

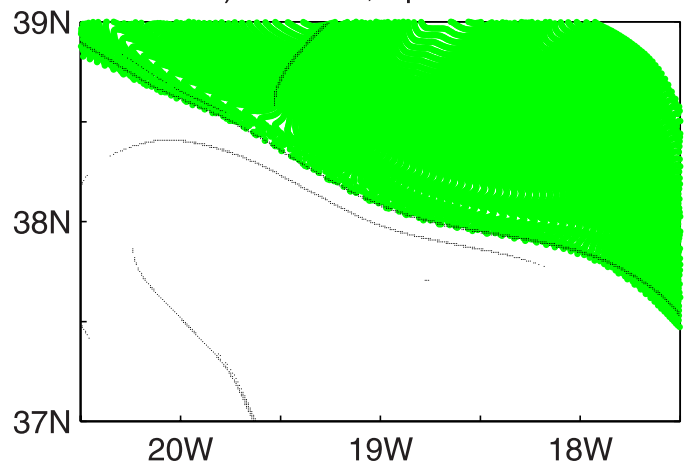

b) Chlorophyll, April 252001

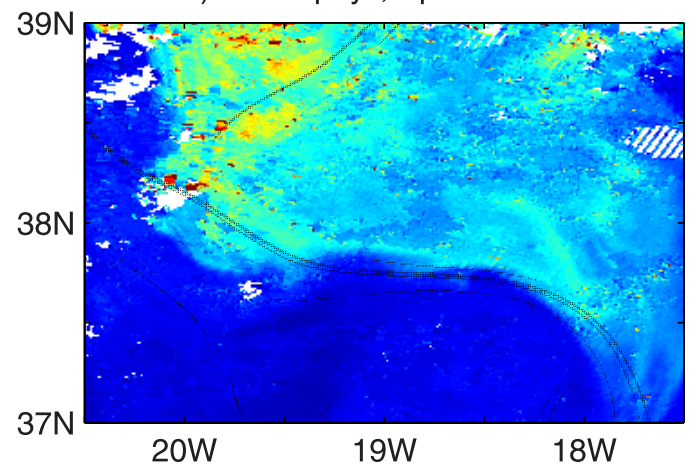

d) SST, April 252001

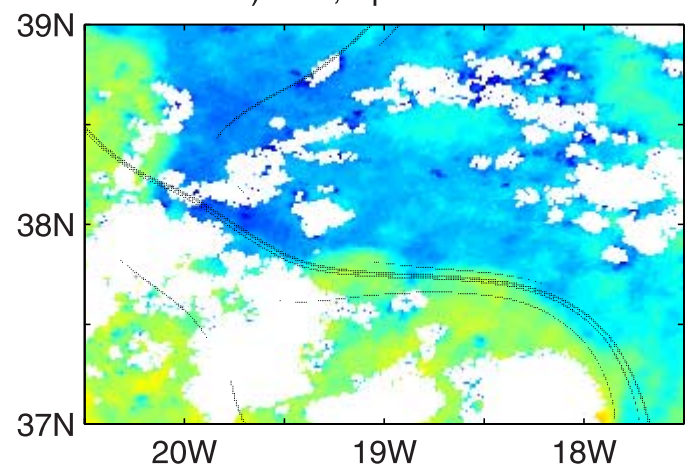

f) Particles, April 252001

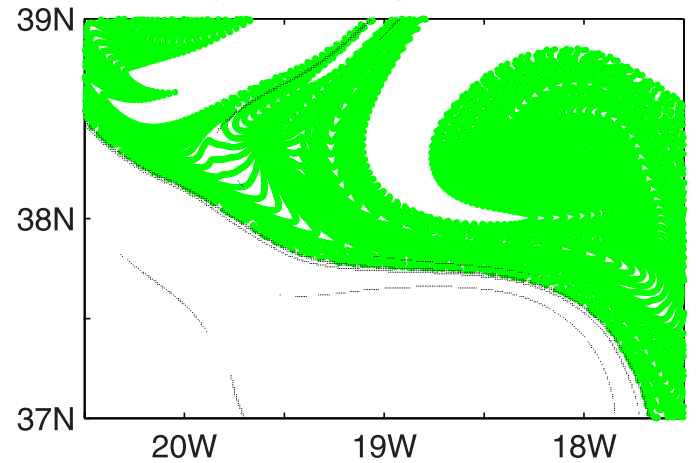

Figure 9. (a, b) SeaWiFS chlorophyll (colors, same scale as in Figure 7f); (c, d) AVHRR SST (colors). (e, f) Time evolution of synthetic passive particles (green dots) advected by the geostrophic velocities. The particles are initiated on 26 March 2001 as a meridional front at $38.5^{\circ} \mathrm{N}$. The snapshots are from consecutive days 2 weeks apart. The black lines correspond to the strong unstable manifolds.

dependency of the velocity field. This leads to the formation of an impermeable kernel, as discussed in section 3.2.

\subsection{Chlorophyll Filaments}

[32] Lastly, we examine the particular case of a chlorophyll filament located over a hyperbolic point (Figure 11). In this location, the flow field exhibits a large strain that stretches passive tracers along the unstable manifold and contracts it along the stable manifold (Figures 11a and 11b). Consequently, the chlorophyll distribution forms a filament along the unstable manifold (Figure 11c). This raises the question of the source of the initial positive chlorophyll anomaly that is being stretched. At first glance, from the geostrophic velocities (Figure 11d), it seems that this filament is not fed by the large chlorophyll values found in the northwest corner, because the velocities are directed toward the northwest. The filament is not inside a lobe of the unstable manifold, that is a mechanism by which an intrusion against the instantaneous velocity field is possible (see Figure 5a). We are thus left with two explanations. The first is intermittent Ekman transport acting against the geostrophic flow. Ekman transport can displace a patch of chlorophyll over the unstable manifold, generating a filament by the mechanism demonstrated in Figure $5 \mathrm{c}$. Indeed, this scenario is supported by ECMWF wind stress analysis (Figure 11e), that shows strong wind activity in the days before the formation of the filament. The second explanation for the filament formation is the mechanism discussed 
a) Particles, May 291999

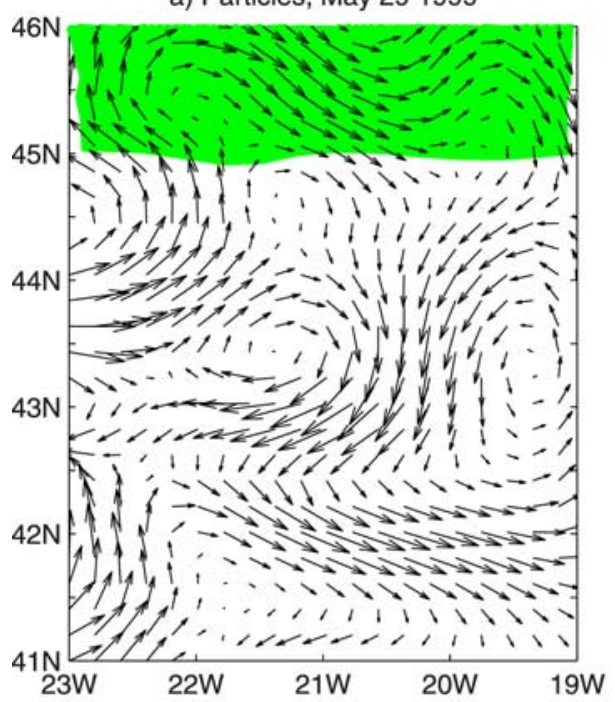

c) Particles, July 71999

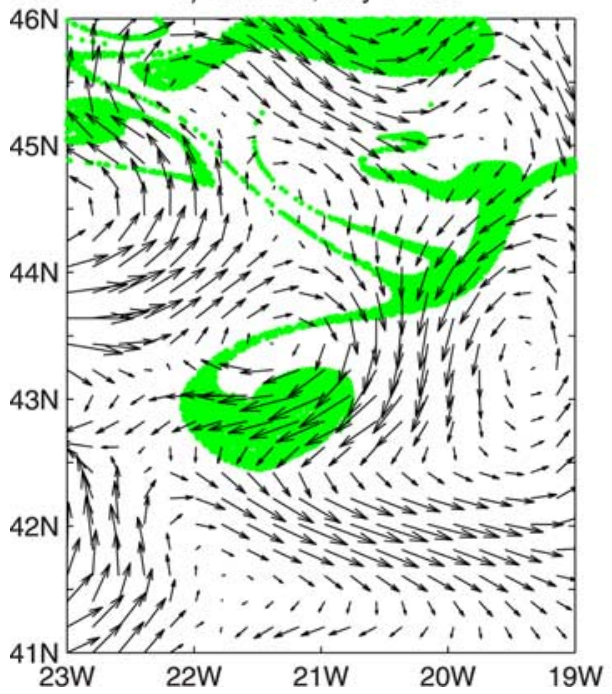

b) Particles, June 171999

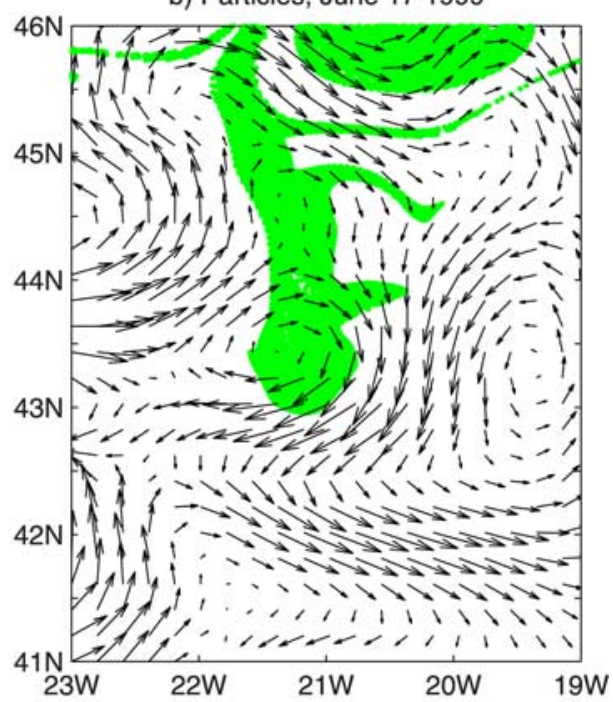

d) Chlorophyll, July 71999

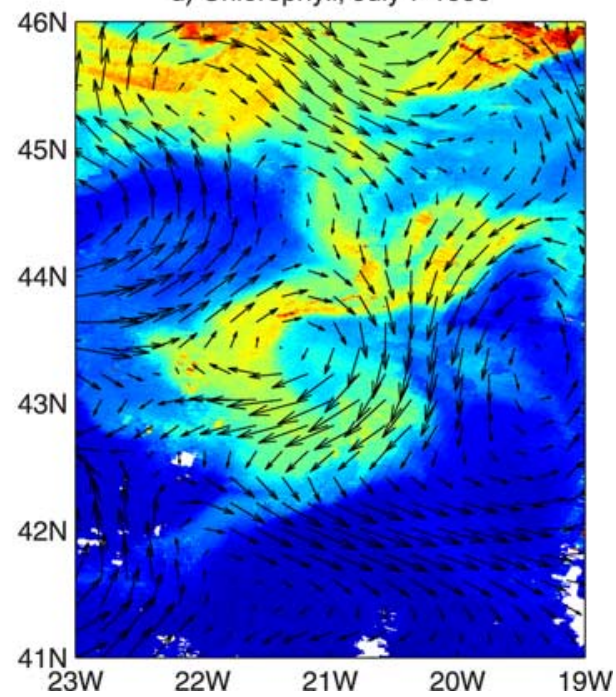

Figure 10. $(\mathrm{a}-\mathrm{c})$ Superposition of strong unstable manifolds (black lines), geostrophic velocities (arrows), and synthetic passive particles advected by the geostrophic velocity field (green dots). The three plots show the evolution of a synthetic large-scale front interacting with a mesoscale eddy over 40 days. The particles are initiated as meridional front at $45^{\circ} \mathrm{N}$. (d) Chlorophyll (colors, same scale as in Figure 7f) and geostrophic velocities (arrows).

in section 3.3, i.e., frontogenesis and ageostrophic circulation in the region of an hyperbolic point, with consequent local formation of chlorophyll by submesoscale upwelling of nutrients along the unstable manifold. This scenario is supported by SST data (Figure 11f), that show strong temperature gradients in the same region of the filament. An equation for estimating the vertical velocity $w$ at a depth of $200 \mathrm{~m}$ in the northeast Atlantic from the density anomalies is proposed by Legal et al. [2006]: $w=$ $-250 \Delta \rho \mathrm{m} /$ days where $\Delta \rho$ is the density difference over a typical lengthscale of $10 \mathrm{~km}$. Figure $11 \mathrm{f}$ shows a thermal gradient of about $0.5^{\circ} \mathrm{C}$. Assuming typical thermal expansion coefficients of the order of $0^{\circ}-0.2^{\circ} \mathrm{C}$, we get to an estimation of $w$ in the range of $25 \mathrm{~m} /$ day, corresponding to an energetic vertical cell capable of nutrient upwelling. We conclude that both Ekman transport and frontogenesis contributed to the filament formation. This is a typical case in which both mechanisms suggested in section 3.3 act together for the formation of the same pattern.

\section{Discussion}

[33] In this study, satellite data were used to investigate how the large-scale phytoplankton spring bloom in the northeast Atlantic is modulated by the mesoscale dynamics. Despite the region being an area of low EKE, mesoscale stirring by the geostrophic currents is found to generate mesoscale and submesoscale chlorophyll structures.

[34] Here we used two independent satellite data sets: chlorophyll and SLA. From the latter, the geostrophic velocities are derived. From the velocity field, we use the FSLE technique to locate the unstable manifolds of the 
a) Particles, June 252001

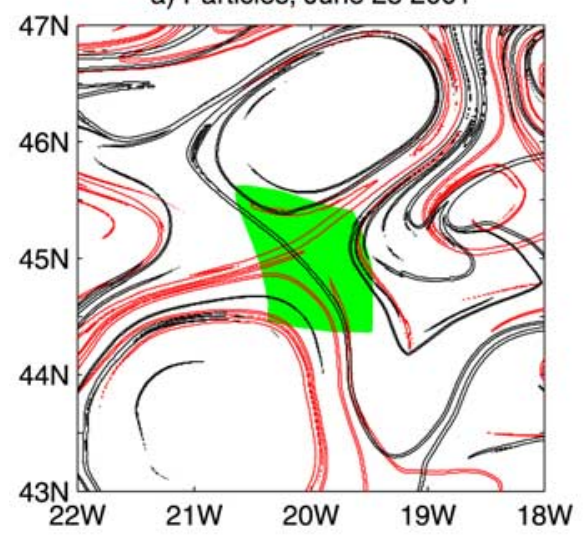

c) Chlorophyll

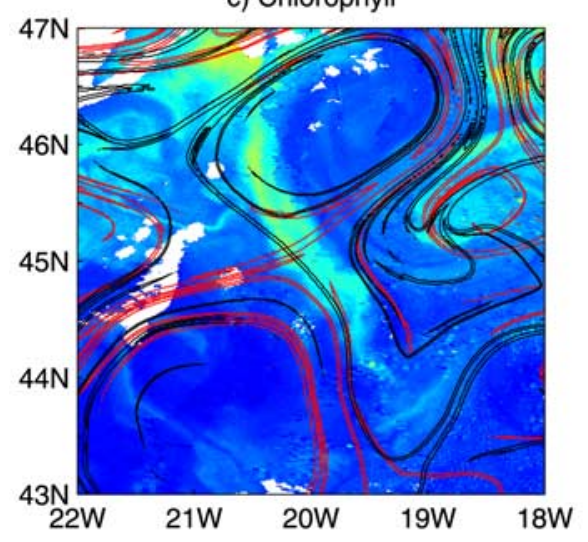

e) Wind

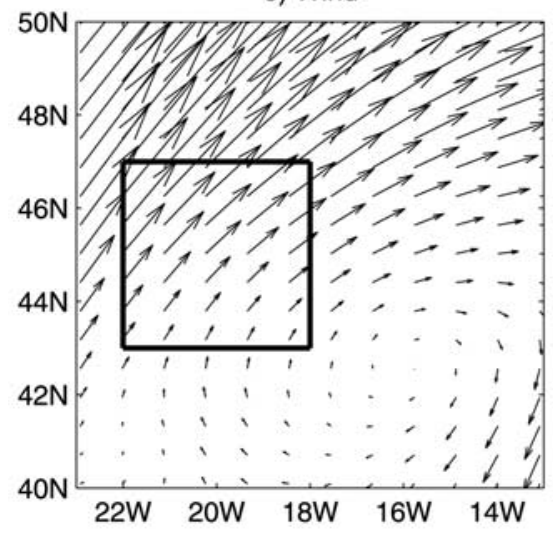

b) Particles, June 302001

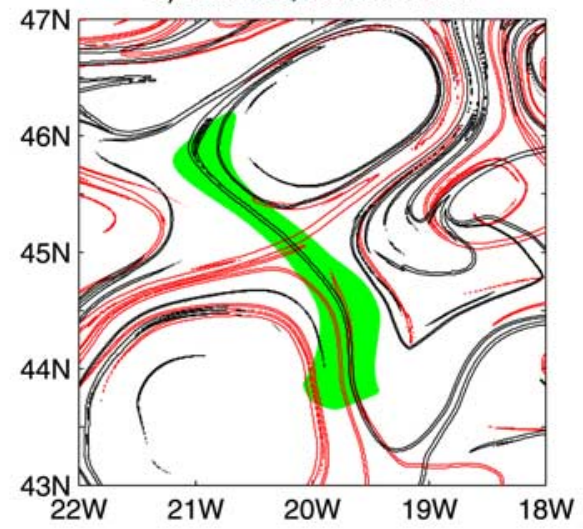

d) Geostrophic velocities
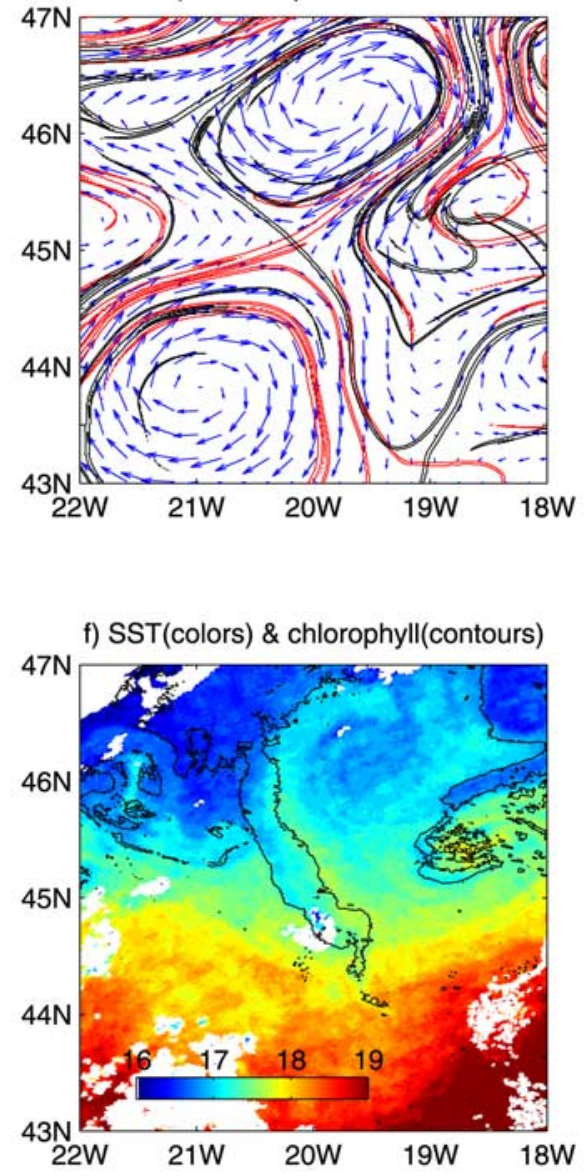

Figure 11. (a, b) Time evolution of synthetic passive particles (green dots) advected by the geostrophic velocity field. The particles are initiated on 24 June 2001 as a patch around the hyperbolic point at $20^{\circ} \mathrm{W} /$ $45^{\circ} \mathrm{N}$; (c) chlorophyll (colors, same scale as in Figure 7f); (d) geostrophic velocity field (arrows); (e) wind stress (arrows, the frame marks the boundaries of the other images in this figure); and (f) superposition of chlorophyll (contours) and SST (colors). The data are from 30 June 2001. The red and black lines correspond respectively to the strong stable and unstable manifolds.

Lagrangian chaotic flow. These manifolds are expected to act as strong sticking barriers for the $2 \mathrm{D}$ advection of passive tracers, and indeed, we found good agreement between the manifolds and the chlorophyll structures, indicating that $2 \mathrm{D}$ stirring is responsible to a large extent for the formation of mesoscale chlorophyll patterns.

[35] The formation of small-scale structures in tracer's distribution by horizontal stirring requires an initial source 
of spatial heterogeneity. In most cases observed here, the source of heterogeneity is the large-scale meridional chlorophyll gradient associated with the northward propagation of the northeast Atlantic bloom. In these cases, the formation of mesoscale and submesoscale patterns result from the direct cascade of the chlorophyll variance injected at large scale. While this process has been simulated with an idealized 2D-turbulence model including the evolution of biological tracers [Abraham, 1998], this work provides, to the best of our knowledge, the first observational evidence of this cascade process.

[36] The cascade appears as a modulation of the largescale chlorophyll front and in intrusions of chlorophyll-rich structures (either eddies or filaments) from the north to the south (or inversely, intrusions of chlorophyll poor structures from the south to the north). These intrusions reach up to $3^{\circ}$ in latitude and act as an efficient diffusive mechanism for the large scale. The importance of this diffusion mechanism mediated by mesoscale eddies has been brought up for idealized tracers and nutrients in eddy-resolving experiments [Lee et al., 1997; Lee and Williams, 2000].

[37] An interesting finding is that the 2D cascade of the initial large-scale gradient can create chlorophyll anomalies within mesoscale eddies (both cyclonic and anticyclonic). The proposed mechanism involves water exchange between the interior of the eddy and its surroundings, and is made possible by the chaotic stirring and mixing, stemmed by the time-dependent nature of the velocity field. This mechanism also explains the formation of spirals within eddies. The inner core of the eddy is protected against mixing between the interior and the exterior. Note that this is also consistent with floats and subsurface water masses analysis from POMME field measurements. The protection of the inner core explains the small spots of high chlorophyll observed on some of the images: they are not mixed with the surrounding poorer waters. The formation of positive chlorophyll anomalies within eddies through 2D cascade were shown in recent model studies [Lévy and Klein, 2004; Lévy, 2003]. Another commonly referred mechanism to explain positive chlorophyll anomalies within eddies is the "eddy pumping" which involves a vertical transport of nutrients through the doming of isopycnals [McGillicuddy et al., 1998]. Here, comparing the data with the simulation of purely 2D passive tracers evolving from a large-scale front, we show that the observed positive anomalies can result from the $2 \mathrm{D}$ cascade. This is in agreement with $U z$ and Yoder [2004], who, on the basis of the relationship between mesoscale anomalies of satellitederived chlorophyll and SST, found that the most important influence of mesoscale motion on the distribution of chlorophyll is advection of the existing gradients.

[38] Our results suggest that chlorophyll variance is also injected at small scales within specific areas of the flow associated with hyperbolic points as local events of upwelling. This mechanism is supported by recent numerical studies, which show that vertical motions within stirring regions can have a significant impact on the vertical injection of any tracer characterized by a strong vertical gradient [Mahadevan and Archer, 2000; Lévy et al., 2001; Martin et al., 2002; Lévy and Klein, 2004].

[39] Note that another potential importance of these vertical motions is their effect on the restratification of the upper layers of the ocean [Lapeyre et al., 2006]. The effect can be dominant in prebloom conditions as shown by the numerical study of Lévy et al. [2005a] for the POMME area, but is negligible in bloom and postbloom conditions.

[40] On the basis of the comparison with chlorophyll images, we deduce that application of the FSLE method on satellite altimetry data is adequate for the study of mesoscale patterns and in some cases even for submesoscale transport processes. The FSLE method is shown to be a useful tool to identify transport barriers and hence to affect chlorophyll pattern formation. The fact that the area is characterized by rather low EKE values suggests that the method would be even more efficient in a region of high EKE.

[41] There are three main limitations to the approach described here. The first limitation is the relatively low resolution (in space and in time) of the altimetric data from which we derive the geostrophic velocities. While the Lagrangian analysis allows us to detect submesoscale filaments coming from the chaotic stirring, some other smallscale features are clearly not resolved (see for instance the small meandering of the chlorophyll and SST fronts in Figure 9). In some cases the low time resolution in altimetry poses a difficulty in accurately localizing even mesoscale structures when the geostrophic field evolves faster than the 1 -week sampling rate. The second limitation is that, when estimating the surface velocity from altimetry we did not take into account quantitatively ageostrophic components, such as the secondary circulation described qualitatively in Figure 6 or the Ekman transport associated with the wind. Finally, in this study we do not take into account the impact of biological activity that is known to have an important role in modulating plankton distribution at the mesoscale [López et al., 2001; Martin et al., 2002; Srokosz et al., 2003]. These limitations explain some of the mismatch that sometimes appears between fronts and manifolds.

[42] The fact that both horizontal transport and vertical upwelling act in phase, strengthening chlorophyll fronts over unstable manifolds, is an advantage for our analysis, supporting the correlation between chlorophyll fronts and manifolds for both postbloom and locally produced chlorophyll. On the other hand, the same argument shows that passive horizontal advection and vertical production are intrinsically entangled, and that it is not possible to resolve the effect of the two by an analysis of chlorophyll and geostrophic velocities images alone. A natural extension of our results is therefore the integration of a Lagrangian analysis with biogeochemical models and with vertical velocity estimation by density anomalies and wind stress. A similar combined approach should be able to attempt a quantitative prediction of chlorophyll gradients and a parameterization of transport on plankton bloom.

\section{Appendix A: Manifold Detection by Lyapunov Exponent Calculation}

[43] Let us come back to Figure 4a, that is the typical situation one has in mind when probing a field with Lyapunov exponent calculation. A tracer initialized in the vicinity of the stable manifold (green dots) is advected toward the equilibrium point where it is subjected to the stretching effect of the unstable manifold. Conversely, for a tracer initialized in a region dominated by recirculation, the 
stretching effect is smaller. The simplest way for quantifying the stretching is to consider the maximum growth rate among two points in the tracer volume. For the case of the green points, the growth is given by the exponential expansion due to the unstable manifold. Calling the initial separation $\delta_{0}$, the separation after time $\tau \delta_{\tau}$, and the positive eigenvalue of the equilibrium $\lambda$, the growth is expressed by

$$
\delta_{\tau}=\delta_{0} e^{\lambda \tau}
$$

[44] By inverting equation (A1), one can obtain $\lambda$ from the tracer experiment of Figure $4 \mathrm{a}$,

$$
\lambda=\frac{1}{\tau} \log \left(\delta_{\tau} / \delta_{0}\right)
$$

[45] Equation (A2) in fact defines a way for probing the space and looking for the stable manifolds of strong equilibrium points. At any point (longitude, latitude) and at any time $t$, we can initialize a volume (that is a surface for a $2 \mathrm{D}$ case), advect it, and by using equation (A2) we can measure a growth rate $\lambda$. All volumes initialized over the stable manifold go toward the equilibrium point where they experience the same stretching and thus (except for a small transient) provide the same value for $\lambda$. The stable manifolds appear in the $\lambda$ field as lines of local maxima. The unstable manifolds can be also detected with the same algorithm, by integrating the velocity field backward in time. Equation (A2) corresponds to the Lyapunov exponent in the limits $\delta_{0} \rightarrow 0$ and $\tau \rightarrow \infty$. For finite values of $\delta_{0}$ and $\tau$ we get instead the so called finite-time or finite-size Lyapunov exponents. The two methods depend on the way that is used to set the time of integration $\tau$. One possibility (finite-time Lyapunov exponent) is to choose $\tau$ a priori, using the same value for all the points; another possibility (finite-size Lyapunov exponent) is to prescribe $\tau$ implicitly, setting the value of $\delta_{\tau}$, and finding for each initial condition the time $\tau$ when points initially separated by $\delta_{0}$ reach a final distance $\delta_{\tau}$. From a theoretical viewpoint finite-size and finite-time Lyapunov exponents are quite different. Moreover, by scanning a velocity field in $\delta_{0}$, the finite-size Lyapunov exponents provides a powerful tool for probing a turbulent field at different scale [see, e.g., Artale et al., 1997]. However, for the use that we make here (the detection of the manifolds), they are similar. The FSLEs are slightly better for our application, in the sense that they allow to tune the time of integration depending on the local properties of the field. In fact there is a trade-off for the choice of $\tau$ : a large time allows to obtain a more precise value for $\lambda$ (by weighting more the stretching in the vicinity of the equilibrium point) but can also be more affected by the time variation of equilibrium and manifolds. The FSLEs allow to use a small integration time whenever a strong hyperbolic structure is encountered, since in this case the final separation $\delta_{\tau}$ is reached faster.

[46] Acknowledgments. This study was supported by the Centre National d'Etudes Spatiales (CNES) and MERCATOR. F. d'O. is a Marie-Curie fellow, grant 024717-DEMETRA. Carine Lesage is thanked for processing of SeaWiFS data and Youssef Amar for processing of AVHRR data. Eric Greiner and Marie-Helene Rio are acknowledged for providing the RIO-5 MSLH MDT. E. H. and Y. L. are grateful to the Porter School of Environmental Studies at Tel-Aviv University (grant
0603413101). Special thanks go to the Goddard Space Flight Center (GSFC/NASA) for providing the SeaWiFS images used in this work. The altimeter products were produced by Ssalto/Duacs and distributed by Aviso, with support from CNES.

\section{References}

Abraham, E. R. (1998), The generation of plankton patchiness by turbulent stirring, Nature, 391, 577-580.

Abraham, E. R., and M. M. Bowen (2002), Chaotic stirring by a mesoscale surface-ocean flow, Chaos, 12, 373-381.

Abraham, E. R., C. S. Law, P. W. Boyd, S. J. Lavender, M. T. Maldonado, and A. Bowie (2000), Importance of stirring in the development of an iron-fertilized phytoplankton bloom, Nature, 407, 727-730.

Arhan, M., A. Colin de Verdiere, and L. Mémery (1994), The eastern boundary of the subtropical North Atlantic, J. Phys. Oceanogr., 24, 1295-1316.

Artale, V., G. Boffetta, A. Celani, M. Cencini, and A. Vulpiani (1997), Dispersion of passive tracers in closed basins: Beyond the diffusion coefficient, Phys. Fluids, 9, 3162-3171.

Assenbaum, M., and G. Reverdin (2005), Near real time analysis of the mesoscale circulation during the POMME experiment, Deep Sea Res., Part I, 30, 2343-2353.

Aurell, E., G. Boffetta, A. Crisanti, G. Paladin, and A. Vulpiani (1997), Predictability in the large: An extension of the Lyapunov exponent, J. Phys. A Math Gen., 30, 1-26.

Boffetta, G., G. Lacorata, G. Redaelli, and A. Vulpiani (2001), Detecting barriers to transport: A review of different techniques, Physica D, 159, 58-70.

de Boyer-Montegut, C., G. Madec, A. S. Fischer, A. Lazar, and D. Iudicone (2004), Mixed layer depth over the global ocean: An examination of profile data and a profile based climatology, J. Geophys. Res., 109, C12003, doi:10.1029/2004JC002378.

d'Ovidio, F., V. Fernández, E. Hernández-Garciá, and C. López (2004), Mixing structures in the Mediterranean sea from Finite-Size Lyapunov Exponents, Geophys. Res. Lett., 31, L17203, doi:10.1029/ 2004GL020328.

Esaias, W. E., G. C. Feldman, C. R. McLain, and J. A. Elord (1986), Monthly satellite-derived phytoplankton pigment distribution for the North Atlantic ocean basin, Eos Trans. AGU, 67, 835-837.

Follows, M., and S. Dutkiewicz (2002), Meteoroligcal modulations of the North Atlantic spring bloom, Deep Sea Res., Part II, 49, 321-344.

Hakim, G. J., C. Snyder, and D. J. Muraki (2002), A new surface model for cyclone-anticyclone asymetry, J. Atmos. Sci., 59, 2405-2420.

Haller, G., and G. Yuan (2000), Lagrangian coherent structures by mixing in two-dimesional turbulence, Physica D, 147, 352-370.

Koh, T., and B. Legras (2002), Hyperbolic lines and the stratospheric polar vortex, Chaos, 12, 382-394.

Lapeyre, G., and P. Klein (2006), Impact of the small-scale elongated filaments on the oceanic vertical pump, J. Mar. Res., 64, 835-851.

Lapeyre, G., P. Klein, and B. L. Hua (2006), Oceanic restratification forced by surface frontogenesis, J. Phys. Oceanogr., 36, doi:10.1175/ JPO2923.11577.

Le Cann, B., M. Assnbaum, J.-C. Gascard, and G. Reverdin (2005), Observed mean and mesoscale upper ocean circulation in the midlatitude northeast Atlantic, J. Geophys. Res., 110, C07S05, doi:10.1029/ 2004JC002768.

Lee, M.-M., and R. G. Williams (2000), The role of eddies in the isopycnic transfer of nutrients and their impact on biological production, J. Mar. Res., 38, 895-917.

Lee, M.-M., D. P. Marshall, and R. G. Williams (1997), On the eddy transfer of tracers: Advective or diffusive?, J. Mar. Res., 55, 483-505.

Legal, C., P. Klein, A.-M. Treguier, and J. Paillet (2006), Diagnosis of the vertical motions in a mesoscale stirring region, J. Phys. Oceanogr., 37(5), 1413-1424, doi:10.1175/JPO3053.1.

Lévy, M. (2003), Mesoscale variability of phytoplankton and of new production: Impact of the large-scale nutrients distribution, J. Geophys. Res., 108(C11), 3358, doi:10.1029/2002JC001577.

Lévy, M., and P. Klein (2004), Does the low frequency variability of mesoscale dynamics explain the a part of the phytoplankton and zooplankton spectral variability, Proc. R. Soc., Ser. A, 460, 1673-1687.

Lévy, M., P. Klein, and A.-M. Treguier (2001), Impact of sub-mesoscale physics on production and subduction of phytoplankton in an oligotrophic regime, J. Mar. Res., 59, 535-565.

Lévy, M. M. Gavart, L. Mémery, G. Caniaux, and A. Paci (2005a), A fourdimensional map of the spring bloom in the northeast Atlantic (POMME experiment): Results of a prognostic model, J. Geophys. Res., 110, C07S21, doi:10.1029/2004JC002588

Lévy, M., Y. Lehahn, J.-M. Andre, L. Mémery, H. Loisel, and E. Heifetz (2005b), Production regimes in the northeast Atlantic: A study based on A study based on Sea-viewing Wide Field-of-view Sensor (SeaWiFS) chlorophyll and OGCM mixed layer depth, J. Geophys. Res., 110, C07S10, doi:10.1029/2004JC002771. 
López, C., Z. Neufeld, Z. Hernández-Garciá, and P. H. Haynes (2001), Chaotic advection of reacting substances: Plankton dynamics on meandering jet, Phys. Chem. Earth, Part B, 26, 313

Mahadevan, A., and D. Archer (2000), Modeling the impact of fronts and mesoscale circulation on the nutrient supply and biogeochemistry of the upper ocean, J. Geophys. Res., 105, 1209-1225.

Mancho, A. M., D. Small, and S. Wiggins (2004), Computation of hyerbolic trajectories and their stable and unstable manifolds for oceanic flows represented as data sets, Nonlinear Processes Geophys., 11, 17-33.

Martin, A. (2003), Phytoplankton patchiness: The role of lateral stirring and mixing, Prog. Oceanogr., 57, 125-174

Martin, A., K. J. Richards, A. Bracco, and A. Provenzale (2002), Patchy productivity in the open ocean, Global Biogeochem. Cycles, 16(2), 1025, doi:10.1029/2001GB001449.

McGillicuddy, D. J., A. R. Robinson, D. A. Siegal, H. W. Jannasch, R. Johnson, T. D. Dicky, J. Mcneil, A. F. Michaels, and A. H. Knap (1998), Influence of mesoscale eddies on new production in the Sargasso Sea, Nature, 394, 263-265.

Mémery, L., G. Reverdin, J. Paillet, and A. Oschlies (2005), Introduction to the POMME spacial section: Thermocline ventilation and biogeochemical tracer distribution in the northeast Atlantic ocean and impact of mesoscale dynamics, J. Geophys. Res., 110, C07S01, doi:10.1029/2005JC002976.

Ott, E. (1993), Chaos in Dynamical Systems, Cambridge Univ. Press, New York.

Paci, A., G. Caniaux, M. Gavart, H. Giordani, M. Lévy, L. Prieur, and G. Reverdin (2005), A high-resolution simulation of the ocean during the POMME experiment: 1 . Simulation results and comparison with observations, J. Geophys. Res., 110, C07S09, doi:10.1029/2004JC002712.

Paillet, J., and M. Arhan (1996a), Oceanic ventilation in the eastern North Atlantic, J. Phys. Oceanogr., 26, 2036-2052.

Paillet, J., and M. Arhan (1996b), Shallow picnoclines and mode water subduction in the eastern North Atlantic, J. Phys. Oceanogr., 26, 96-114

Pasquero, C. (2005), Differential eddy diffusion of biogeochemical tracers, Geophys. Res. Lett., 32, L17603, doi:10.1029/2005GL023662.

Pedlosky, J. (1987), Geophysical Fluid Dynamics, Springer, New York.

Rio, M.-E., and F. Hernández (2004), A mean dynamic topography computed over the world ocean from altimetry, in-situ measurements and a geoid model, J. Geophys. Res., 109, C12032, doi:10.1029/ 2003JC002226.
Robinson, A. R., et al. (1993). Mesoscale and upper ocean variabilities during the 1989 JGOFS bloom study, Deep Sea Res., Part II, 40, 9-35.

Srokosz, M. A., A. P. Martin, and M. J. R. Fasham (2003), On the role of biological dynamics in plankton patchiness at the mesoscale: An example from the eastern North Atlantic Ocean, J. Mar. Res., 61, 517-537.

Stammer, D., C. Wunsch, and K. Ueyoshi (2006), Temporal changes in ocean eddy transpot, J. Phys. Oceanogr., 36, 543-550.

Sverdrup, H. U. (1953), On conditions for the vernal blooming of phytoplankton, J. Cons. Cons. Int. Explor. Mer, 18, 287-295.

Toner, M., A. D. Kirwan, A. C. Poje, L. H. Kantha, F. E. Muller-Karger, and C. K. R. T. Jones (2003), Chlorophyll dispersal by eddy-eddy interactions in the Gulf of Mexico, J. Geophys. Res., 108(C4), 3105, doi:10.1029/2002JC001499.

Uz, B. M., and J. A. Yoder (2004), High frequency and mesoscale variability in SeaWiFS chlorophyll imagery and its relation to other remotely sensed oceanographic variables, Deep Sea Res., Part II, 51, 1001-1017.

Waugh, D. W., E. R. Abraham, and M. M. Bowen (2006), Spatial variations of stirring in the surface ocean: A case study of the Tasman Sea, J. Phys. Oceanogr., 36, 526-542.

Wiggins, S. (2005), The dynamical systems approach to Lagrangian transport in oceanic flows, Annu. Rev. Fluid Mech., 37, 295-298.

Williams, R. G., and M. J. Follows (2003), Physical transport of nutrients and the maintenance of biological production, in Ocean Biogeochemistry: The Role of the Ocean Carbon Cycle in Global Change, Springer, New York.

Williams, R. G., A. J. McLaren, and M. J. Follows (2000), Estimating of convective supply of nitrate and implied variability in export production in the North Atlantic, Global Biogeochem. Cycles, 14, 1299-1313.

F. d'Ovidio, UMR 8539, Laboratoire de Météorologie Dynamique, Ecole Normale Supérieure, 24 rue Lhomond, F-75231 Paris, France. (dovidio@ lmd.ens.fr)

E. Heifetz, Department of Geophysics and Planetary Sciences, Tel Aviv University, Tel Aviv, Israel. (eyalh@post.tau.ac.il)

Y. Lehahn and M. Lévy, Institut Pierre-Simon Laplace, Laboratoire d'Océanographie et du Climat: Expérimentations et Approches Numériques, Paris, France. (yoav.lehahn@lodyc.jussieu.fr; marina.levy@lodyc. jussieu.fr) 